\title{
Synthesis of Some Biologically Important 3-0xacepham Derivatives
}

\section{Mohammad Rafiqul Islam ${ }^{1}$, Mohammad Nurnabi ${ }^{1}$, A. M. Sarwaruddin Chowdhury $^{1}$, and Mohammad Mehdi Masud ${ }^{2}$}

\author{
${ }^{1}$ Department of Applied Chemistry \& Chemical Technology, University of Dhaka, Dhaka-1000, Bangladesh \\ ${ }^{2}$ Department of Pharmaceutical Chemistry, University of Dhaka, Dhaka-1000, Bangladesh
}

\begin{abstract}
The $6 H$-oxathiazines 1a-e having imine moiety underwent $[2+2]$ cycloaddition with phenoxyacetylchloride in the presence of $\mathrm{Et}_{3} \mathrm{~N}$ to give $\beta$-lactam derivatives 2a-e in high yield. The X-ray crystallographic analysis revealed the relative stereochemistry that the substituents at $C-2$ and $C-4$ were cis configurated. The subtituents at $C-6$ and $C-7$ were also cis to each other. However, the $6 H$-oxathiazines 1f-i containing tert-butyl or methyl group at $C-4$ did not undergo the cycloaddition.
\end{abstract}

Key words: Azetedinone, $\beta$-lactam, oxacepham, cycloaddition, imine, ketene, oxathiazine.

\section{INTRODUCTION}

Even more than 70 years after the discovery of penicillin, $\beta$-lactam-containing antibiotics are still in use for the treatment of infectious diseases caused by various pathogens. However, within a short time after introduction of a new antibiotic, bacterial strains become resistant to it due to the indiscriminate use and as result the antibiotics loose their activities. Moreover, the resistant bacterial strains are affecting the humans with severe damaging effects. Thus a continued effort is needed to fight the infectious diseases by extending the effectiveness of the currently available antibiotics.

The biological activity exhibited by $\beta$-lactam antibiotics is found to be associated with the $\beta$-lactam ring, the reactivity of which in turn is depend on the tail end as well as on the head of the antibiotic molecule. $^{1}$ Modification of the tail end led to the

Correspondence to: Mohammad Nurnabi Tel: 88029661920-73/7395

Email:nnabi@univdhaka.edu

Dhaka Univ. J. Pharm. Sci. 5(1-2): 47-51, 2006 (June-December) introduction of a large number of clinically useful penam and cepham derivatives. ${ }^{2}$ Modification of the head of the antibiotic molecule involved replacement of sulfur atom of these bicyclic compounds by carbon, ${ }^{2}$ nitrogen, ${ }^{3}$ and oxygen ${ }^{4-6}$ in order to enhance the reactivity of the azetedinone carbonyl function and consequently antibacterial activity. It is well accepted that the replacement of ' $S$ ' of the cepham ring with an electronegative atom such as ' $\mathrm{O}$ ' increases the penetration of the molecule through the cell wall of the bacteria due to the greater hydrophilicity and thus imparts the greater activity. ${ }^{7}$ Moreover, some studies showed that the oxacepham derivatives have better $\beta$-lactamase inhibitory activity, especially against cephalosporinase than the ceohem derivatives. ${ }^{8}$ Some reports ${ }^{9,10}$ described the synthesis of cepham derivatives containing nitrogen atom in place of $\mathrm{C}-2$ of the cepham nucleus. It was documented earlier that the introduction of $\mathrm{N}, \mathrm{O}$ atoms in place of $\mathrm{C}-3$ activated the $\mathrm{C}=\mathrm{O}$ group of the $\beta$-lactam ring through inductive effect of the highly electronegative heteroatoms. ${ }^{11}$ However, to the best of our knowledge, only a single report on the 
synthesis of cepham derivatives containing oxygen as third heteroatom in the head portion of the cepham derivative $^{11}$ has been disclosed. Therefore, synthesis of a wide variety of 3-oxacepham derivatives containing fluorine atom in the aromatic ring would deliver more potent drug candidates against pathogenic microorganisms.

Herein, a synthesis and detailed structural elucidation of some biologically potential 3oxacepham derivatives are documented.

\section{MATERIALS AND METHODS}

General. All substances and reagents were commercially available and were used without further purification. Flash column chromatography was performed on Merck silica gel 60 (230-400 mesh). Melting points were determined on a Buchi 535 micro-melting point apparatus and are uncorrected. Microanalyses were obtained using a Yanagimoto CHN recorder MT-5. Proton nuclear magnetic resonance $\left({ }^{1} \mathrm{H}\right.$ NMR) spectra experiments were determined at $400 \mathrm{MHz}$. on a Bruker AC-400P spectrometer. Chemical shifts are reported in parts per million $(\delta)$ downfield from tetramethylsilane (TMS) as internal standard and coupling constants are given in Hertz $(\mathrm{Hz}) .{ }^{13} \mathrm{C}$ NMR spectra were recorded with a Bruker AC-400P $(75 \mathrm{MHz})$ and chemical shift values were reported in parts per million (ppm) relative to $\mathrm{CDCl}_{3}(\delta=77.0)$. Mass spectra were obtained on a Hitachi M-2000 mass spectrometer using electron impact (EI) ionization at $70 \mathrm{eV}$. Infrared spectra were recorded on a JASCO FT/IR-7300 spectrometer either by $\mathrm{KBr}$ pressed disks method or film (neat) method.

General procedure. To a stirred solution of 1 (1 $\mathrm{mmol})$ and triethylamine $(1.5 \mathrm{mmol})$ in anhydrous dichloromethane $(25 \mathrm{ml})$ at $0^{\circ} \mathrm{C}$, was added the phenoxyacetylchloride $(1.45 \mathrm{mmol})$ in anhydrous dichloromethane $(10 \mathrm{ml})$ dropwise during $10 \mathrm{~min}$. The stirring was continued for $2 \mathrm{hrs}$ at $0-5^{\circ} \mathrm{C}$ and then at room temperature for overnight. The reaction mixture was successively washed with water, $10 \%$ aq. sodium bicarbonate and water. The organic layer was separated, dried $\left(\mathrm{Na}_{2} \mathrm{SO}_{4}\right)$ and concentrated to afford the $\beta$-lactam derivatives 2 . The compounds were purified by column chromatography.

2,4-Dimethyl-7-phenoxy-6-phenyl-3-oxa-5thia-1-aza-bicyclo[4.2.0]octan-8-one2a): Colourless crystals (84\%), mp 95-96 ${ }^{\circ} \mathrm{C}$ (Found: $\mathrm{C}, 66.97 ; \mathrm{H}$, 5.56; $\mathrm{N}, 4.10 ; \mathrm{C}_{19} \mathrm{H}_{19} \mathrm{NO}_{3} \mathrm{~S}$ requires $\mathrm{C}, 66.84 ; \mathrm{H}$, $5.61 ; \mathrm{N}, 4.10 \%) ; \delta_{\mathrm{H}}\left(400 \mathrm{MHz}, \mathrm{CDCl}_{3}\right) 1.42(3 \mathrm{H}, \mathrm{d}$, $\left.\mathrm{J}=6.0, \mathrm{CH}_{3}\right), 1.55\left(3 \mathrm{H}, \mathrm{d}, \mathrm{J}=6.0, \mathrm{CH}_{3}\right), 5.18(1 \mathrm{H}, \mathrm{q}$, $\mathrm{J}=6.0, \mathrm{H}-2), 5.61(1 \mathrm{H}, \mathrm{s}, \mathrm{H}-7), 5.72(1 \mathrm{H}, \mathrm{q}, \mathrm{J}=6.0, \mathrm{H}-$ 4), $6.61(2 \mathrm{H}, \mathrm{d}, \mathrm{J}=7.9, \operatorname{Ar}-\mathrm{H}), 6.86(1 \mathrm{H}, \mathrm{t}, \mathrm{J}=7.3, \operatorname{Ar}-$ H), 7.08-7.12 (2H, m, Ar-H), 7.19-7.25 (3H, m, Ar$\mathrm{H}), 7.43-7.45(2 \mathrm{H}, \mathrm{m}, \mathrm{Ar}-\mathrm{H}) ; \delta_{\mathrm{C}}\left(100 \mathrm{MHz}, \mathrm{CDCl}_{3}\right)$ $21.1\left(\mathrm{CH}_{3}\right), 21.6\left(\mathrm{CH}_{3}\right), 70.1(\mathrm{C}-6), 75.4(\mathrm{C}-4), 79.5$ (C-2), 93.5 (C-7), 115.3 (Ar-C), 122.2 (Ar-C), 127.4 (Ar-C), 127.5 (Ar-C), 128.3 (Ar-C), 129.2 (Ar-C), 136.9 (Ar-C), 156.2 (Ar-C) and $165.3(\mathrm{C}=\mathrm{O}) ; \mathrm{m} / \mathrm{z}$ (EI) $341\left(\mathrm{M}^{+}, 2 \%\right)$ and $121 ; v_{\text {max }} / \mathrm{cm}^{-1}(\mathrm{KBr}) 2902$, 1778, 1160, 1496, 1335 and 1116.

6-(4-Chloro-phenyl)-2,4-dimethyl-7-phenoxy3-oxa-5-thia-1-aza-bicyclo[4.2.0]octan-8-one(2b):

Colourless crystals $(87 \%)$, mp $116-117^{\circ} \mathrm{C}$ (Found: $\mathrm{C}$, 60.76; H, 4.59; N, 3.64; $\mathrm{C}_{19} \mathrm{H}_{18} \mathrm{ClNO}_{3} \mathrm{~S}$ requires $\mathrm{C}$, $60.71 ; \mathrm{H}, 4.83 ; \mathrm{N}, 3.73 \%) ; \delta_{\mathrm{H}}\left(400 \mathrm{MHz}, \mathrm{CDCl}_{3}\right)$ $1.39\left(3 \mathrm{H}, \mathrm{d}, \mathrm{J}=6.0, \mathrm{CH}_{3}\right), 1.55\left(3 \mathrm{H}, \mathrm{d}, \mathrm{J}=6.0, \mathrm{CH}_{3}\right)$, $5.15(1 \mathrm{H}, \mathrm{q}, \mathrm{J}=6.0, \mathrm{H}-4), 5.56(1 \mathrm{H}, \mathrm{s}, \mathrm{H}-2), 5.69(1 \mathrm{H}$, q, J=6.0, H-6), $6.63(2 \mathrm{H}, \mathrm{d}, \mathrm{J}=7.3$, Ar-H), 7.11-7.15 $(3 \mathrm{H}, \mathrm{m}, \mathrm{Ar}-\mathrm{H}), 7.21(2 \mathrm{H}, \mathrm{d}, \mathrm{J}=7.3, \operatorname{Ar}-\mathrm{H}), 7.41(2 \mathrm{H}$, d, J=7.3, Ar-H); $\delta_{\mathrm{C}}\left(100 \mathrm{MHz}, \mathrm{CDCl}_{3}\right) 21.2\left(\mathrm{CH}_{3}\right)$, $21.6\left(\mathrm{CH}_{3}\right), 69.4$ (C-6), 75.6 (C-4), 79.7 (C-2), 93.3 (C-7), 115.2 (Ar-C), 122.3 (Ar-C), 127.7 (Ar-C), 129.0 (Ar-C), 129.3 (Ar-C), 134.4 (Ar-C), 135.6 (ArC), $155.9(\mathrm{Ar}-\mathrm{C})$ and $165.0(\mathrm{C}=\mathrm{O}) ; \mathrm{m} / z(\mathrm{EI}) 375\left(\mathrm{M}^{+}\right.$, $6 \%$ ) and 155.0; $v_{\max } / \mathrm{cm}^{-1}(\mathrm{KBr}) 2989,1782,1713$, 1599 and 1494.

\section{6-(4-Fluoro-phenyl)-2,4-dimethyl-7-phe-}

noxy-3-oxa-5-thia-1-aza-bicyclo[4.2.0]octan-8-one (2c): Colourless oil (86\%) (Found: C, 63.28; H, 4.67; $\mathrm{N}, 3.79 ; \mathrm{C}_{19} \mathrm{H}_{18} \mathrm{FNO}_{3} \mathrm{~S}$ requires $\mathrm{C}, 63.49 ; \mathrm{H}, 5.05 ; \mathrm{N}$, $3.90 \%) ; \delta_{\mathrm{H}}\left(400 \mathrm{MHz}, \mathrm{CDCl}_{3}\right) 1.39(3 \mathrm{H}, \mathrm{d}, \mathrm{J}=5.9$, $\left.\mathrm{CH}_{3}\right), 1.55\left(3 \mathrm{H}, \mathrm{d}, \mathrm{J}=5.9, \mathrm{CH}_{3}\right), 5.15(1 \mathrm{H}, \mathrm{q}, \mathrm{J}=5.9$, H-4), $5.59(1 \mathrm{H}, \mathrm{s}, \mathrm{H}-7), 5.68(1 \mathrm{H}, \mathrm{q}, \mathrm{J}=5.9, \mathrm{H}-2)$, $6.62(2 \mathrm{H}, \mathrm{d}, \mathrm{J}=7.9, \mathrm{Ar}-\mathrm{H}), 6.86-6.93(3 \mathrm{H}, \mathrm{m}, \mathrm{Ar}-\mathrm{H})$, $7.11(2 \mathrm{H}, \mathrm{t}, \mathrm{J}=7.9, \mathrm{Ar}-\mathrm{H}), 7.43-7.46(2 \mathrm{H}, \mathrm{m}, \mathrm{Ar}-\mathrm{H})$; 
$\delta_{\mathrm{C}}\left(100 \mathrm{MHz}, \mathrm{CDCl}_{3}\right) 19.6\left(\mathrm{CH}_{3}\right), 23.1\left(\mathrm{CH}_{3}\right), 64.8$ (C-6), 70.4 (C-4), 72.8 (C-2), 96.2 (C-7), 114.2 (ArC), 115.4 (Ar-C), 120.1 (Ar-C), 129.1 (Ar-C), 130.5 (Ar-C), 133.9 (Ar-C), 158.8 (Ar-C), 160.5 (Ar-C) and $173.6(\mathrm{C}=\mathrm{O}) ; m / z(\mathrm{EI}) 359\left(\mathrm{M}^{+}, 6 \%\right)$ and 138.0.

2,4-Diisopropyl-7-phenoxy-6-phenyl-3-oxa-5thia-1-aza-bicyclo[4.2.0]octan-8-one (2d): Pale yellow oil (76\%) (Found: C, 69.50; H, 6.80; N, 3.55; $\mathrm{C}_{23} \mathrm{H}_{27} \mathrm{NO}_{3} \mathrm{~S}$ requires $\mathrm{C}, 69.49 ; \mathrm{H}, 6.85 ; \mathrm{N}, 3.52 \%$ ); $\delta_{\mathrm{H}}\left(400 \mathrm{MHz}, \mathrm{CDCl}_{3}\right) 0.92\left(3 \mathrm{H}, \mathrm{d}, \mathrm{J}=6.0, \mathrm{CH}_{3}\right), 1.03$ $\left(3 \mathrm{H}, \mathrm{d}, \mathrm{J}=6.0, \mathrm{CH}_{3}\right), 1.04\left(3 \mathrm{H}, \mathrm{d}, \mathrm{J}=5.9, \mathrm{CH}_{3}\right), 1.08$ $\left(3 \mathrm{H}, \mathrm{d}, \mathrm{J}=5.9, \mathrm{CH}_{3}\right), 1.75\left(1 \mathrm{H}, \mathrm{m},-\mathrm{CH}-\mathrm{Me}_{2}\right), 2.04$ $\left(1 \mathrm{H}, \mathrm{m},-\mathrm{CH}-\mathrm{Me}_{2}\right), 4.75(1 \mathrm{H}, \mathrm{d}, \mathrm{J}=6.0, \mathrm{H}-4), 4.90$ $(1 \mathrm{H}, \mathrm{d}, \mathrm{J}=5.9, \mathrm{H}-2), 5.55(1 \mathrm{H}, \mathrm{s}, \mathrm{H}-7), 6.60(2 \mathrm{H}, \mathrm{d}$, $\mathrm{J}=7.3$, Ar-H), 7.07-7.7.11 (3H, m, Ar-H), 7.17-7.24 $(3 \mathrm{H}, \mathrm{m}, \mathrm{Ar}-\mathrm{H}), 7.52(2 \mathrm{H}, \mathrm{d}, \mathrm{J}=7.3, \mathrm{Ar}-\mathrm{H}) ; \delta_{\mathrm{C}}(100$ $\left.\mathrm{MHz}, \mathrm{CDCl}_{3}\right)$ 13.6 $\left(\mathrm{CH}_{3}\right), 14.0\left(\mathrm{CH}_{3}\right), 14.6\left(\mathrm{CH}_{3}\right)$, $16.1\left(\mathrm{CH}_{3}\right), 36.7\left(\mathrm{CH}-\mathrm{Me}_{2}\right), 40.2\left(\mathrm{CH}-\mathrm{Me}_{2}\right), 65.4(\mathrm{C}-$ 6), 73.9 (C-4), 76.3 (C-2), 96.2 (C-7), 114.2 (Ar-C), 120.1 (Ar-C), 126.9 (Ar-C), 128.4 (Ar-C), 128.9 (ArC), 129.1 (Ar-C), 138.3 (Ar-C), 158.8 (Ar-C) and $173.6(\mathrm{C}=\mathrm{O}) ; \mathrm{m} / \mathrm{z}(\mathrm{ES}) 397.2\left(\mathrm{M}^{+}, 100 \%\right)$.

2,4-Dibutyl-7-phenoxy-6-phenyl-3-oxa-5-thia1-aza-bicyclo[4.2.0]octan-8-one (2e): Colourless oil (83\%) (Found: C, 70.85; H, 7.37; N, 3.39; $\mathrm{C}_{25} \mathrm{H}_{31} \mathrm{NO}_{3} \mathrm{~S}$ requires $\mathrm{C}, 70.55 ; \mathrm{H}, 7.34 ; \mathrm{N}, 3.29 \%$ ); $\delta_{\mathrm{H}}\left(400 \mathrm{MHz}, \mathrm{CDCl}_{3}\right) 0.96\left(3 \mathrm{H}, \mathrm{t}, \mathrm{J}=5.8, \mathrm{CH}_{3}\right), 1.10$ $\left(3 \mathrm{H}, \mathrm{t}, \mathrm{J}=5.8, \mathrm{CH}_{3}\right), 1.33\left(2 \mathrm{H}, \mathrm{m},-\mathrm{CH}_{2}-\right), 1.28(2 \mathrm{H}$, $\left.\mathrm{m},-\mathrm{CH}_{2}-\right), 1.31\left(2 \mathrm{H}, \mathrm{m},-\mathrm{CH}_{2}-\right), 1.40\left(2 \mathrm{H}, \mathrm{m},-\mathrm{CH}_{2}-\right)$, $1.79\left(2 \mathrm{H}, \mathrm{m},-\mathrm{CH}_{2}-\right), 1.82\left(2 \mathrm{H}, \mathrm{m},-\mathrm{CH}_{2}-\right), 3.88(1 \mathrm{H}$, $\mathrm{t}, \mathrm{J}=6.0, \mathrm{H}-4), 4.94(1 \mathrm{H}, \mathrm{t}, \mathrm{J}=6.0, \mathrm{H}-2), 5.67(1 \mathrm{H}, \mathrm{s}$, H-7), $6.61(2 \mathrm{H}, \mathrm{d}, \mathrm{J}=7.9$, Ar-H), $6.86(1 \mathrm{H}, \mathrm{t}, \mathrm{J}=7.3$, Ar-H), 7.08-7.12 (2H, m, Ar-H), 7.19-7.25 (3H, m, Ar-H), 7.43-7.45 (2H, m, Ar-H); $\delta_{\mathrm{C}}(100 \mathrm{MHz}$, $\left.\mathrm{CDCl}_{3}\right) 14.0\left(\mathrm{CH}_{3}\right), 22.7\left(\mathrm{CH}_{3}\right), 23.1\left(-\mathrm{CH}_{2}-\right), 24.0(-$ $\left.\mathrm{CH}_{2}-\right), 25.5\left(-\mathrm{CH}_{2}-\right), 26.3\left(-\mathrm{CH}_{2}-\right), 34.2\left(-\mathrm{CH}_{2}-\right), 37.7$ (- $\left.\mathrm{CH}_{2}-\right), 65.4$ (C-6), 74.2 (C-4), 76.6 (C-2), 96.2 (C7), 114.2 (Ar-C), 120.1 (Ar-C), 126.9 (Ar-C), 128.4 (Ar-C), 128.9 (Ar-C), 129.1 (Ar-C), 138.3 (Ar-C), $158.8(\mathrm{Ar}-\mathrm{C})$ and $173.6(\mathrm{C}=\mathrm{O}) ; \mathrm{m} / z(\mathrm{EI}) 397\left(\mathrm{M}^{+}\right.$$\mathrm{CO}, 3 \%$ ) and 77 .

\section{RESULTS AND DISCUSSION}

The synthesis of $6 H$-oxathiazines 1 have been previously reported ${ }^{12}$ and in this study we employed the $6 H$-oxathiazines 1 having imine moiety to react with phenoxyacetylchloride in the presence of $\mathrm{Et}_{3} \mathrm{~N}$ to give $\beta$-lactam derivatives 2 in high yields. From the mechanistic point of view, deprotonation of the $\alpha$ proton of phenoxyacetylchloride afforded phenoxyketene intermediate, which underwent a $[2+2]$ cycloaddition with the imine moiety of the oxathiazine $\mathbf{1}$ to afford the $\beta$-lactam derivatives 2 (Scheme 1). To examine the versatility of the methodology a wide variety of $6 \mathrm{H}-1,3,5$-oxathiazines were examined for the generality, scope and limitation of this approach (Table 1).

However, the [2+2] cycloaddition of 1f-i with ketene did not proceed to afford the expected products. It was found that in the substrates $\mathbf{1}$, substituents $\left(\mathrm{R}^{1}\right.$ and $\left.\mathrm{R}^{2}\right)$ at $\mathrm{C}-2$, $\mathrm{C}-4$ and $\mathrm{C}-6$ positions played vital role for the reaction. When the $R^{2}$ substituent was a bulky group ( $t$-butyl), the reaction did not occur (Table 1, entry 7-9). When both $\mathrm{R}^{1}$ and $\mathrm{R}^{2}$ substituents were methyl group, the reaction was also unsuccessful (Table 1, entry 6), which might be due to the electron enrichment at imine moiety by methyl group $\left(\mathrm{R}^{1}\right)$, which in turn deactivated the imine moiety toward [2+2] cycloaddition with ketene.

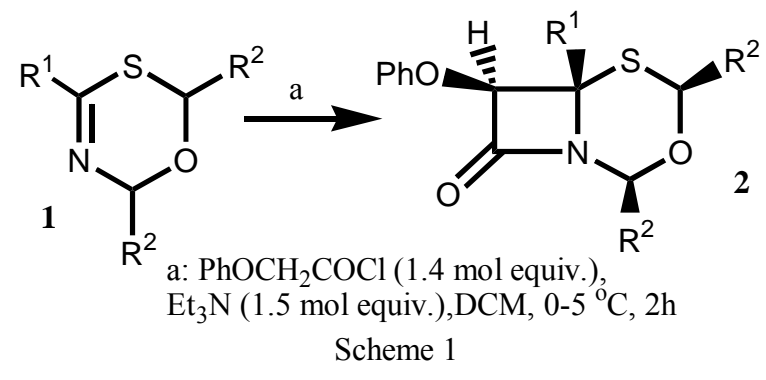

The relative stereochemistry of all the stereogenic centers were determined by X-ray analysis of 2a, and the ORTEP drawing of $\mathbf{2 a}$ is shown in Figure $1 .{ }^{13}$ The X-ray crystallographic analysis revealed that the orientation of substituents at $C-2$ and $C-4$ were $c i s$ to each other. The substituents at $C-6$ and $C-7$ were also cis to each other 
Table 1. Synthesis of 3-oxacepham 2

\begin{tabular}{|c|c|c|c|c|c|}
\hline Entry & Substrate & $\mathrm{R}^{1}$ & $\mathrm{R}^{2}$ & product & yield $(\%)^{a}$ \\
\hline 1 & $1 \mathrm{a}$ & $\mathrm{Ph}$ & $\mathrm{Me}$ & $2 \mathrm{a}$ & 84 \\
\hline 2 & $1 b$ & $p-\mathrm{Cl}-\mathrm{C}_{6} \mathrm{H}_{4}$ & $\mathrm{Me}$ & $2 b$ & 87 \\
\hline 3 & $1 \mathrm{c}$ & $p-\mathrm{F}-\mathrm{C}_{6} \mathrm{H}_{4}$ & $\mathrm{Me}$ & $2 \mathrm{c}$ & 86 \\
\hline 4 & $1 d$ & $\mathrm{Ph}$ & $i$-Pr & $2 d$ & 76 \\
\hline 5 & $1 \mathrm{e}$ & $\mathrm{Ph}$ & $n-\mathrm{C}_{4} \mathrm{H}_{9}$ & $2 \mathrm{e}$ & $83^{b}$ \\
\hline 6 & $1 \mathrm{f}$ & $\mathrm{Me}$ & $\mathrm{Me}$ & - & 0 \\
\hline 7 & $1 \mathrm{~g}$ & $\mathrm{Ph}$ & $t-\mathrm{C}_{4} \mathrm{H}_{9}$ & - & 0 \\
\hline 8 & $1 \mathrm{~h}$ & $\mathrm{Me}$ & $t-\mathrm{C}_{4} \mathrm{H}_{9}$ & - & 0 \\
\hline 9 & $1 \mathrm{i}$ & $p-\mathrm{Cl}-\mathrm{C}_{6} \mathrm{H}_{4}$ & $t-\mathrm{C}_{4} \mathrm{H}_{9}$ & - & 0 \\
\hline
\end{tabular}

${ }^{\mathrm{a}}$ Isolated yield, ${ }^{\mathrm{b}}$ Diastereomeric mixture (20:1)

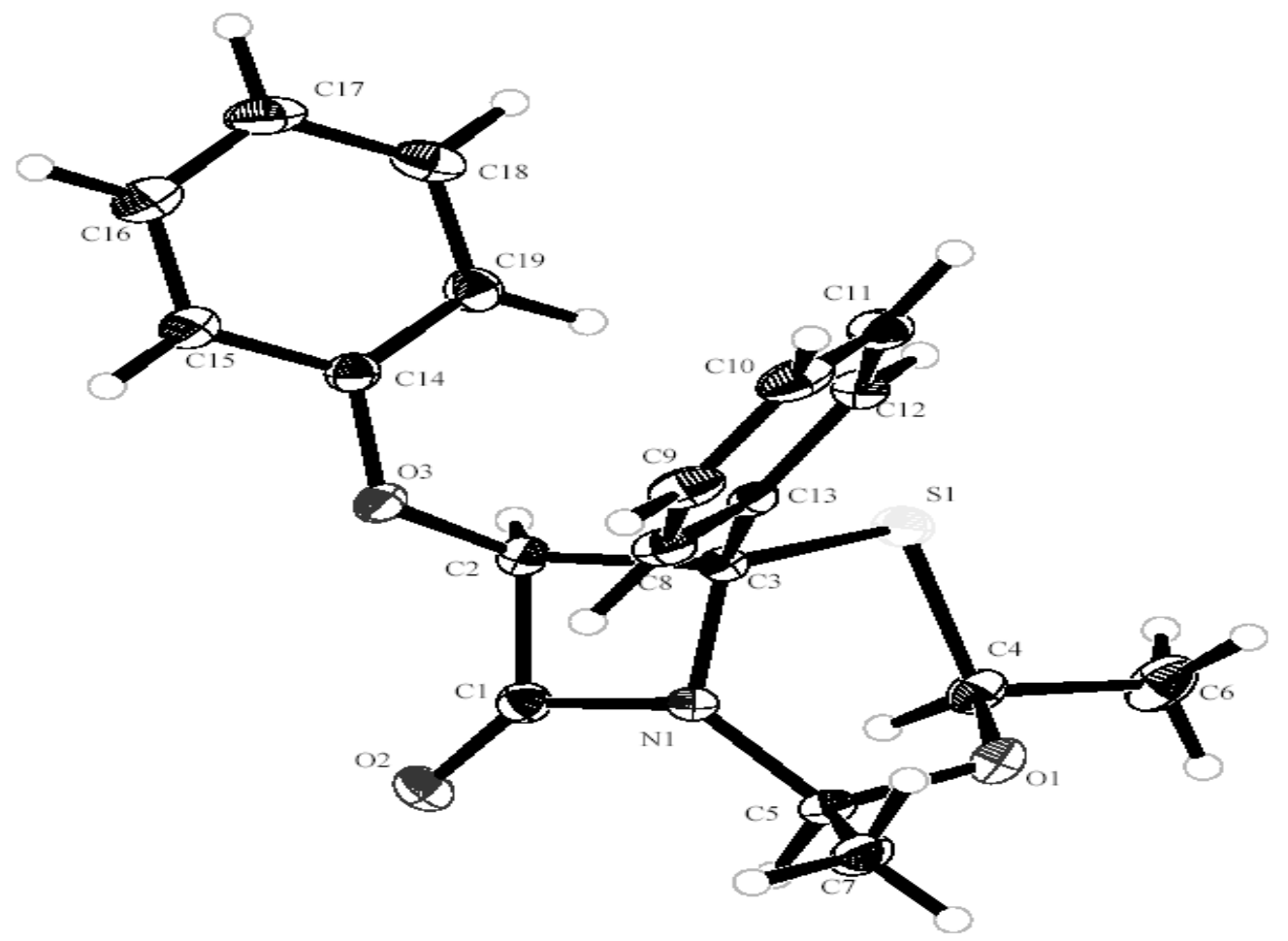

Figure 1. ORTEP Drawing of compound 2a: Selected bond lengths (A) and Angles (deg): O1-C4=1.413(2); S1-C3=1.838(2); S1$\mathrm{C} 4=1.839(2) ; \quad \mathrm{O} 2-\mathrm{C} 1=1.210(2) ; \mathrm{O} 3-\mathrm{C} 14=1.379(2) ; \mathrm{N} 1-\mathrm{C} 3=1.474(2) ; \mathrm{O} 1-\mathrm{C} 5=1.425(2) ; \mathrm{O} 3-\mathrm{C} 2=1.399(2) ; \mathrm{N} 1-\mathrm{C} 1=1.365(2) ; \mathrm{N} 1-$ $\mathrm{C} 5=1.457(2) ; \mathrm{C} 3-\mathrm{S} 1-\mathrm{C} 4=98.08(8) ; \mathrm{C} 2-\mathrm{O} 3-\mathrm{C} 14=116.8(1) ; \mathrm{C} 1-\mathrm{N} 1-\mathrm{C} 5=128.9(1) ; \mathrm{O} 2-\mathrm{C} 1-\mathrm{N} 1=131.6(2) ; \mathrm{S} 1-\mathrm{C} 3-\mathrm{N} 1=110.2(1) ; \mathrm{S} 1-\mathrm{C} 3-$ $\mathrm{C} 13=11113.7(1) ; \mathrm{N} 1-\mathrm{C} 3-\mathrm{C} 13=115.6(1)$.

\section{CONCLUSION}

A wide variety of biologically important 3oxacepham derivatives have been synthesized through [2+2] cycloaddition of the imine moiety of $6 H$-oxathiazines with phenoxyacetylchloride in the presence of $\mathrm{Et}_{3} \mathrm{~N}$ to give $\beta$-lactam derivatives in high yields. The scope and limitation of this approach was also explored.

\section{REFERENCES}

1. Manhas, M.S. and Bose, A.K. 1971. Beta lactams-Natural and synthetic: Part I. Wiley Interscience, New York, p.187.

2. Ratcliffe, R.W., Salzmann, T.N. and Christensen, B.G. 2000. A novel synthesis of the carbapen-2-em-ring system. Tetrahedron Lett. 21, 31-34.

3. Brown, D., Brown, G.A., Andrews, M., Large, J.M., Urban, D., Butts, C.P., Hales, N.J. and Gallagher, M. 2002. The azomethine ylide strategy for $\beta$-lactam synthesis. Azapenams and 1-azacephams. J. Chem. Soc., Perkin Trans. 1, 20142021. 
4. Hamashima, Y., Yamamoto, S., Kubota, T., Tokura, K., Ishikura, K., Minami, K., Matsubara, F., Yamaguchi, M., Fikkawa, I. and Nagata, W. 1979. Synthesis studies on $\beta$ lactam antibiotics. 19. Synthesis of 3'-nor-type 1oxacephems. Tetrahedron Lett. 20, 4947-4950.

5. Howarth, T.T., Brown, A.G. and King, T.J. 1976. Clavulanic acid, a novel $\beta$-lactam isolated from Streptomyces clavuligerus; X-ray crystal structure analysis. Chem. Commun. 266b.

6. Furman, B., Thurmer, R., Kaluza, Z., Volter, W. and Chmielewski, M. 1999. A new acetal resin valuable for the solid-phase synthesis of 1-oxacepham via a cyclization/ cleavage step. Tetrahedron Lett. 40, 5909-5912.

7. Korolkovas, A. 1988. Essentials of medicinal Chemistry. $2^{\text {nd }}$ Ed., John Wiley and Sons, NewYork, p. 783.

8. Nishimura, S., Sasaki, H., Yasuda, N., Matsumoto, Y., Kamimura, T., Sakane, K. and Takaya, T. 1989. Synthesis and biological activity of 7 alpha-hydroxyethyl-1-oxacephem derivatives. J. Antibiot. (Tokyo) 42, 1124-1132.

9. Herak, J.J., Vinkovic, M. and Lukic, I. 1995. Functional Derivatives of 4-Oxoazatidine-2-sulfinic Acids in Asymetric Synthesis of 2-Azacepham Sulfoxides and their Transformation. Tetrahedron 51, 5083-5092.
10. Herak, J.J., Vinkovic, M., Mandic, Z., Lukic, I., Tomic, M. and Kovacevic, M. 1994. Synthesis, Structural Characterization and Stereocontrolled Degradation of 2Azacephams. Tetrahedron Assym. 5, 1605-1612.

11. Kar, G.K., Chatterjee, G.G. and Ray, J.K. 1988. Studies on New $\beta$-lactams: Synthesis of 3-Oxacepham Derivatives. Ind. J. Chem. 27B, 786-789.

12. Islam, M.R,, Kazuaki, S., Shigenobu, A., Yuji, T. and Chizuko, K. 2004. Novel Conversion of $6 H-1,3,5-$ Oxathiazine $S$-Oxides into 5-Membered Heterocyclic Compounds. Heteroatom Chem. 15, 175-186.

13. X-ray crystallographic data for 2a. Colorless prism of 2a suitable for X-ray investigation was obtained from ether. Crystal data: $\mathrm{C}_{19} \mathrm{H}_{19} \mathrm{NO}_{3} \mathrm{~S}, \quad \mathrm{FW}=341.42$, crystal size $0.25 \times 0.20 \times 0.20 \mathrm{~mm}^{3}$, monoclinic, space group $\mathrm{P} 2{ }_{1} / \mathrm{n}(\# 14)$, $a=12.393(4), \quad b=8.449(3), \quad c=16.687(6) \quad \AA, \quad \beta=99.908(5)^{\circ}$, $\mathrm{V}=1721.0(1) \AA^{3}, \mathrm{Z}=4, D_{\text {calc }}=1.317 \mathrm{~g} / \mathrm{cm}^{3}, \mu=2.04 \mathrm{~cm}^{-1}$. From 16063 reflections measured 3814 were unique $\left(\mathrm{R}_{\mathrm{int}}=0.025\right)$. $\mathrm{R}=0.036, \mathrm{R}_{\mathrm{w}}=0.037, \mathrm{MoK} \alpha\left(\lambda=0.71070 \AA, \mathrm{T}=-100.0^{\circ} \mathrm{C}\right.$. The structure was solved by direct methods (SIR92). 